\title{
A VARIATIONAL APPROACH TO A CLASS OF NONLINEAR EIGENVALUE PROBLEMS
}

\author{
PETER HESS ${ }^{1}$
}

\begin{abstract}
Aвstract. Let $f$ be a real-valued differentiable function defined on the real reflexive Banach space $X$. The problem of minimizing $f$ over a subset of $X$ is investigated under the following mild monotonicity assumption on the derivative $f^{\prime}$ of $f:$ if $\left\{u_{n}\right\}$ is a sequence in $X$ converging weakly to some $u \in X$, then $\lim \sup \left(f^{\prime} u_{n}, u_{n}-u\right)$ $\geqq 0$. The eigenvalue problem $f^{\prime} u=\lambda g^{\prime} u$ for some $\lambda \in R^{1}$, with $g^{\prime}$ being the derivative of a further function $g$, is then reduced to that first question.
\end{abstract}

Introduction. We consider the following variational

Problem $\left(^{*}\right)$. To minimize a real-valued function $f$ over a subset $C$ of the real reflexive Banach space $X$.

If the function $f$ is differentiable, a solution of Problem $\left(^{*}\right)$ with $C=X$ yields a solution of the equation $f^{\prime} u=0$. If $f$ is differentiable and $C=M_{c}(g)=\{u \in X: g(u)=c\}$ is a level set of a further differentiable function $g$, a solution of Problem $\left(^{*}\right)$ is (under additional restrictions) a solution of the eigenvalue problem $f^{\prime} u=\lambda g^{\prime} u$ for some real $\lambda$.

For functions $f$ which can be represented in the form $f(u)=\Phi(u, u)$, with $\Phi$ a semiconvex mapping of $X \times X$ into the reals, Problem $\left({ }^{*}\right)$ is treated in Browder [1], where the abstract results are also applied to multiple integral functionals.

In the recent note [6] Browder attacks Problem (*) for differentiable functions $f$ and convex sets $C$, making use of properties of the derivative $f^{\prime}$ rather than of those of $f$. In the application to multiple integral functionals, his new results are, however, weaker than those he got in [1]. Further the convexity assumption on the set $C$ is rather restrictive; eigenvalue problems cannot be studied by this method.

In the present note we investigate Problem $\left({ }^{*}\right)$ for differentiable functions with a related method which does not demand the convexity of $C$. Compared with [6] we require more continuity properties on the derivative of the function $f$, but can considerably weaken the monotonicity assumptions on $f^{\prime}$.

\footnotetext{
Presented to the Society, August 17, 1970; received by the editors August 12, 1970 and, in revised form, October 26, 1970.

AMS 1969 subject classifications. Primary 4780, 4610.

Key words and phrases. Variational problem, differentiable function, reflexive Banach space, operator of monotone type, nonlinear eigenvalue problem, multiple integral functional.

1 This research was partially supported by NSF Grant GP-23563.
} 
In $\$ 1$ we prove an abstract theorem (Theorem 1) on the existence of minima of functions on a subset of a reflexive Banach space. As a consequence we obtain in $\$ 2$ a result on nonlinear eigenvalue problems (Theorem 2) which strengthens corresponding statements in [2], [4], [8] established by approximation methods of Galerkin type.

When applied to multiple integral functionals defined on an appropriate Sobolev space, Theorems 1 and 2 allow one to slightly extend related assertions by Browder [1], [2], [4] and Nečas [10].

1. Let $X$ be a real Banach space, $X^{*}$ its conjugate space. By $(v, u)$ we denote the duality pairing between elements $v \in X^{*}$ and $u \in X$. We use the symbols " $\rightarrow$ " and " $\rightarrow$ " to denote strong and weak convergence, respectively.

Definition. $A$ mapping $T$ of $X$ into $X^{*}$ satisfies condition (P) if for each sequence $\left\{u_{n}\right\}$ in $X$ converging weakly to some $u \in X$, $\lim \sup \left(T u_{n}, u_{n}-u\right) \geqq 0$.

We remark that condition $(\mathrm{P})$ is an extremely mild monotonicity assumption. Indeed, the class of mappings satisfying condition (P) contains most of the operators of monotone type considered so far, as for example monotone mappings, pseudomonotone mappings [3], $[5],[6],[7]$ and demicontinuous operators of type $(S)^{+}[2],[3],[4]$, $[5],[6],[8],[9]$.

If $u$ is a point of $X$, the function $f: X \rightarrow R^{1}$ is said to have the element $w$ of $X^{*}$ as its Fréchet derivative at $u$ if, for each $v \in X$,

$$
f(u+v)=f(u)+(w, v)+\varphi(v)
$$

where $\lim \left\|_{v}\right\|_{\rightarrow 0}(\varphi(v) /\|v\|)=0$. We let $f^{\prime} u$ denote the derivative of $f$ at $u$. If $f$ is differentiable on $X, f^{\prime}$ is a well-defined mapping of $X$ into $X^{*}$.

Our main result is

THEOREM 1. Let $f$ be a real-valued differentiable function defined on the real reflexive Banach space $X$ and $C$ a weakly sequentially closed subset of $X$. Suppose that $\lim \left\|_{u}\right\|_{\rightarrow \infty, u \in C} f(u)=+\infty$, and that the derivative $f^{\prime}$ is continuous and satisfies condition (P). Then $f$ assumes a minimum value on $C$, and for any minimizing sequence $\left\{u_{n}\right\}$ in $C$ with $u_{n} \rightarrow u \in C, f(u)=\inf _{v \in C} f(v)$.

Theorem 1 strengthens the corresponding theorem of Browder [6] in the case that the derivative $f^{\prime}$ is continuous.

Proof of Theorem 1. Let $\left\{u_{n}\right\}$ be a minimizing sequence, i.e. a sequence in $C$ with the property that $f\left(u_{n}\right) \rightarrow \inf _{v \in C} f(v)$. Since $f(u)$ $\rightarrow+\infty$ as $\|u\| \rightarrow \infty, u \in C$, the sequence $\left\{u_{n}\right\}$ is bounded. By the 
reflexivity of the space $X$ and the weak sequential closedness of $C$ we may assume after passing to a subsequence that $\left\{u_{n}\right\}$ converges weakly to some element $u \in C$. We claim that $f(u)=\lim f\left(u_{n}\right)$. Clearly $f(u) \geqq \lim f\left(u_{n}\right)$. Let us suppose that $f(u)>\lim f\left(u_{n}\right)$. By definition of the derivative and the fundamental theorem of the calculus,

$$
f\left(u_{n}\right)-f(u)=\int_{0}^{1}\left(f^{\prime}\left(u+s\left(u_{n}-u\right)\right), u_{n}-u\right) d s .
$$

The mean value theorem of integral calculus implies that, for each $n$,

$$
f\left(u_{n}\right)-f(u)=\left(f^{\prime}\left(u+s_{n}\left(u_{n}-u\right)\right), u_{n}-u\right),
$$

with $s_{n} \in[0,1]$. Let $v_{n}=u+s_{n}\left(u_{n}-u\right)$. Then $v_{n} \rightarrow u$. Since, by assumption, $\lim f\left(u_{n}\right)-f(u)<0$, there exist constants $n_{0}$ and $\lambda>0$ such that

$$
\left(f^{\prime} v_{n}, u_{n}-u\right) \leqq-\lambda<0
$$

for all $n>n_{0}$. By passing to a subsequence, we may further assume that $s_{n} \rightarrow \sigma \in[0,1]$. We now distinguish between two cases:

(i) $\sigma>0$. Then $s_{n} \geqq \epsilon>0$ for a suitable $\epsilon$ and $n$ sufficiently large, and consequently

$$
\left(f^{\prime} v_{n}, v_{n}-u\right)=s_{n}\left(f^{\prime} v_{n}, u_{n}-u\right) \leqq-s_{n} \lambda \leqq-\epsilon \lambda<0
$$

for large $n$, which contradicts property $(\mathrm{P})$ of the mapping $f^{\prime}$.

(ii) $\sigma=0$. In this case $v_{n} \rightarrow u$, and by continuity of $f^{\prime}, f^{\prime} v_{n} \rightarrow f^{\prime} u$. We infer that $\left(f^{\prime} v_{n}, u_{n}-u\right) \rightarrow 0$, in contradiction to (1).

Hence

$$
f(u)=\lim f\left(u_{n}\right)=\inf _{\bullet \in C} f(v) . \quad \text { Q.E.D. }
$$

REMARK. With the same arguments one shows that a continuously differentiable function whose derivative satisfies condition $(P)$ is weakly sequentially lower semicontinuous.

2. The key to the discussion of nonlinear eigenvalue problems by variational methods is the following Lemma, known as Lusternik's principle:

Lemma [1], [4], [11]. Let $X$ be a real Banach space and $f, g$ two continuously differentiable real-valued functions on $X$. Suppose that $g^{\prime} u \neq 0$ for $u$ in the level set $M_{c}(g)=\{u \in X: g(u)=c\}$. If $f(u)$ has a local extremum at a point $u_{0} \in M_{c}(g)$ with respect to $u \in M_{c}(g)$, then there exists $\lambda \in R^{1}$ such that $f^{\prime} u_{0}=\lambda g^{\prime} u_{0}$. 
The following result on a class of nonlinear eigenvalue problems is now an immediate consequence of Theorem 1 and the Lemma.

TheOREM 2. Let $X$ be a real reflexive Banach space and $f, g$ two realvalued functions defined on $X$. Suppose that $g$ is weakly sequentially continuous, and that $f$ and $g$ are continuously differentiable on $X$, with $f^{\prime}$ satisfying condition (P). Assume further that for a given constant $c$ the level set $M_{c}(g)=\{u \in X: g(u)=c\}$ is nonempty, that $g^{\prime} u \neq 0$ for $u \in M_{c}(g)$, and that $f(u) \rightarrow+\infty$ as $\|u\| \rightarrow \infty, u \in M_{c}(g)$. Then $f$ assumes its infimum on the set $M_{c}(g)$ at a point $u_{0}$ which is a solution of the eigenvalue equation $f^{\prime} u_{0}=\lambda g^{\prime} u_{0}$ for some real $\lambda$.

In Theorem 2, the assumption on the weak sequential continuity of $g$ can be replaced by the requirement that $g^{\prime}$ is compact, since also the latter condition implies that the level set $M_{c}(g)$ is weakly sequentially closed. With this alteration, Theorem 2 generalizes corresponding results in [4], [8] obtained by approximation methods and Lagrange's multiplier principle.

ADDED IN PROOF. The observation that condition $\left(^{*}\right)$ is (even in the case of compact operators $T$ ) a proper weakening of the concept of angle-boundedness, permits one to extend some recent results by Amann on Hammerstein equations with compact angle-bounded kernel $T$ and bounded, continuous $N$ (Hammersteinsche Gleichungen mit kompakten Kernen, Math. Ann. 186 (1970), 334-340, Theorem 1; Existence theorems for equations of Hammerstein type (to appear in Applicable Analysis), Theorem 3). In addition, our proof is considerably simpler than those given by Amann.

THEOREM. Let $T$ be a compact linear operator of the real Banach space $X$ into $X^{*}$ satisfying condition

$\left({ }^{*}\right)$ There exists $d>0$ such that $(T u, u) \geqq d\|T u\|^{2}$ for all $u \in X$.

Let further $N$ be a bounded continuous mapping of $X^{*}$ into $X$, and assume that for some function $\varphi: R^{+} \rightarrow R^{+}$satisfying $\varphi(r)=o\left(r^{2}\right)$ as $r \rightarrow+\infty$, we have $(v, N v) \geqq-c\|v\|^{2}-\varphi(\|v\|)$ for $v \in X^{*}$, with $c<d$. Then the equation $w+T N w=0$ admits a solution $w$ in $X^{*}$.

Proof. By the boundedness and continuity of the mapping $N$ and the compactness of $T$, the operator $T N: X^{*} \rightarrow X^{*}$ is continuous and compact. We define a continuous mapping $C_{t} w=C(w, t): X^{*} \times[0,1]$ $\rightarrow X^{*}$, by $C_{t} w=w+t T N w$. We first show that $C_{t} w \neq 0$ for $\|w\|=R$ sufficiently large and $t \in[0,1]$. Indeed, $C_{0} w \neq 0$ for $w \neq 0$. Let thus $0<t \leqq 1$, and suppose $w+t T N w=0$ for some $w \in X^{*}$. Then $0=(w, N w)$ $+t(T N w, N w) \geqq-c\|w\|^{2}-\varphi(\|w\|)+t d\|T N w\|^{2}$. Since $\|T N w\|$ $=t^{-1}\|w\|$, we obtain $\varphi(\|w\|)+c\|w\|\left\|^{2} \geqq t\left(\varphi(\|w\|)+c\|w\|^{2}\right) \geqq d\right\| w \|^{2}$, 
which implies the uniform boundedness of $\|w\|$. Next we observe that the degree theory of Leray-Schauder is applicable to the homotopy $\left\{C_{t}\right\}_{0 \leq t \leq 1}$. Since the degree $\operatorname{deg}\left(C_{0}, B_{R}, 0\right)$ of the mapping $C_{0}=I$ on the open ball $B_{R}$ in $X^{*}$ with respect to the origin is +1 and this degree remains invariant through the homotopy $\left\{C_{t}\right\}_{0 \leqq i \leqq 1}$, we infer that the equation $C_{1} w=w+T N w=0$ admits a solution $w \in B_{R}$. Q.E.D.

\section{REFERENCES}

1. F. E. Browder, Variational methods for nonlinear elliptic eigenvalue problem, Bull. Amer. Math. Soc. 71 (1965), 176-183. MR 31 \#3707.

2. - Nonlinear eigenvalue problems and Galerkin approximations, Bull. Amer. Math. Soc. 74 (1968), 651-656. MR 37 \#2043.

3. - Nonlinear operators and nonlinear equations of evolution in Banach spaces, Proc. Sympos. Pure Math., vol. 18, Part 2, Amer. Math. Soc., Providence, R. I. (to appear).

4. - Existence theorems for nonlinear partial differential equations, Proc. Sympos. Pure Math., vol. 16, Amer. Math. Soc., Providence, R. I., 1970.

5. - Nonlinear elliptic boundary value problems and the generalized topological degree, Bull. Amer. Math. Soc. 76 (1970), 999-1005.

6. - Pseudo-monotone operators and the direct method of the calculus of variations, Arch. Rational Mech. Anal. 38 (1970), 268-277.

7. F. E. Browder and P. Hess, Nonlinear mapping of monotone type in Banach spaces (to appear).

8. P. Hess, Nonlinear functional equations and eigenvalue problems in nonseparable Banach spaces, Comment. Math. Helv. (to appear).

9. - Nonlinear functional equations in Banach spaces and homotopy arguments, Bull. Amer. Math. Soc. 77 (1971), 211-215.

10. J. Nečas, Les équations elliptiques nonlinéaires, Czechoslovak Math. J. (19) 94 (1969), 252-274. MR $40 \# 6045$.

11. M. M. Vainberg, Variational methods for the study of nonlinear operators, GITTL, Moscow, 1956; English Transl., Holden-Day, San Francisco, Calif., 1964. MR 19, 567; MR 31 \#638.

University of Chicago, Chicago, Illinois 60637 